\title{
S-COMPACTNESS VIA IDEALS
}

\author{
M. E. ABD EL-MONSEF, E. F. LASHIEN AND A. A. NASEF
}

\begin{abstract}
Compactness modulo an ideal has not been widely studied. Many new sorts of weak compactness have been introduced to topological spaces in the last twenty years but have not been studied using ideals. So, the main aim of our work is to study relations between ideals and some types of weak compactness. We initiate types of compactness modulo an ideal that generalize semi-compactness, $S$-closeness, $S$-Lindelöfness and other types of compactness. Moreover, we study some of their properties and characterizations.
\end{abstract}

\section{Introduction and Preliminaries}

The concept of compactness modulo an ideal was first introduced by Newcomb [27] in 1967, Rančin [32] in 1972 and studied by Hamlett and Janković [17] in 1990. In this paper, we use the concept of semi-open sets [21] to introduce new sorts of compactness modulo an ideal, namely $S I$-compactness and countably $S I$-compactness. Throughout this note, $(X, \tau)$ and $(Y, \sigma)$ (or simply $X$ and $Y$ ) denote topological spaces on which no separation axiom is assumed unless explicitly stated. Let $W$ be a subset of $X . \mathrm{Cl}_{\tau}(W)\left(\operatorname{resp}\right.$. $\left.\operatorname{Int}_{\tau}(W)\right)$ will denote the closure (resp. interior) of $W$ with respect to $\tau$ (we will omit $\tau$ if there is no possibility of confusion). The complement of $W$ will be denoted by $(X-W)$. A subset $W$ of $X$ is said to be semi-open [21] (resp. preopen [24], $\alpha$-open [28],

Received June 1, 1992.

1980 AMS Subject classification code: 54D30, 54C10

Key words and phrases: I-compact, countably I-compact, SI-compact, countably SIcompact. 
$\beta$-open [1]) if $W \subset \mathrm{Cl}(\operatorname{Int}(W))($ resp. $W \subset \operatorname{Int}(\mathrm{Cl}(W)), W \subset \operatorname{Int}(\mathrm{Cl}(\operatorname{Int}(W)))$, $W \subset \mathrm{Cl}(\operatorname{Int}(W))))$. The complement of semi-open (resp. preopen) is called semi-closed [21] (resp. preclosed [24], The intersection of all semi-closed sets containing $W \subset X$ called semi-closure [12] of $W$ and denoted by $s-\mathrm{Cl}(W)$. Recall that $W$ is said to be regular open (resp. regular closed) if $W=\operatorname{Int}(\mathrm{Cl}(W))$ (resp. $W=\mathrm{Cl}(\operatorname{Int}(W))$ ). $W \subset X$ is said to be regular semi-open (Cameron, 1978 [10]) if there exists a regular open set $U$ of $X$ such that $U \subset W \subset \mathrm{Cl}(U)$. The family of all regular open (resp. regular closed, semi-open, preopen, $\alpha$ open, $\beta$-open) sets of $(X, \tau)$ is denoted by $\operatorname{RO}(X, \tau)(\operatorname{resp} \cdot \operatorname{RC}(X, \tau), \operatorname{SO}(X, \tau)$, $\left.\mathrm{PO}(X, \tau), \tau^{\alpha}, \beta O(X, \tau)\right)$. It is shown in [28] that $\tau^{\alpha}$ is a topology on $X$ and $\tau \subset \tau^{\alpha}$. Given a set $X$, a collection $I$ of subsets of $X$ is called an idcal [20] on $X$ if:

(i) $A \in I$ and $B \subseteq A$, implies $B \in I$ (heredity), and

(ii) $A \in I$ and $B \in I$, implies $A \cup B \in I$ (additivity)

If $X \notin I$, then $I$ is said to be a proper ideal. Observe that in this case $\{A$ : $X-A \in I\}$ is a filter, and hence proper ideals are sometimes called dual filters. We will denote by $(X, \tau, I)$ a nonempty set $X$, a topology $\tau$ on $X$, and an ideal $I$ on $X$.

The following notations will be used in this paper:

$\langle\phi\rangle:$ The simplest ideal on $X$.

$I_{f}$ : The ideal of finite subsets of $X$.

$I_{c}$ : The ideal of countable subsets of $X$.

$I_{n}$ : The ideal of nowhere dense sets in $(X, \tau)$,

$\left(\mathbb{W} \in I_{n}\right.$ iff $\left.\operatorname{Int}(\mathrm{Cl}(W))=\phi\right)$.

$\tau \mid A$ : The relative (or subspace) topology on $A \subseteq X$.

$I \mid A$ : The restriction of $I$ to $A$, i.e.

$I \mid A=\{E \cap A: E \in I\}$. Note that $I \mid A$ is an ideal [17].

$\tau(A)$ : The simple extension of $\tau$ by $A \subseteq X$, where, $\tau(A)=\left\{G \cup\left(G^{\prime} \cap A\right): G, G^{\prime} \in \tau, A \notin \tau\right\}$ [22].

$\tau[A]$ : The local discrete extension of $\tau$ by $A$ where $\tau[A]=\{U-B: U \in \tau, B \subseteq A)$ for each $A \subseteq X .[34]$. 
$\tau(\Im):$ The filter extension of $\tau$ by a filter $\Im$ on $X$ where $\tau(\Im)=\{U \cap F: U \in \tau, F \in \Im\}[6]$.

Given a function $f:(X, \tau) \rightarrow(Y, \sigma), f$ is said to be irresolute [13] (resp. $\beta$-irresolute [23]) if for every semi-open (resp. $\beta$-open) set $B \subseteq Y, f^{-1}(B)$ is semi-open (resp. $\beta$-open) in $X$, and $f$ is said to be pre-semi-open [13] if for every semi-open set $W \subseteq X, f(W)$ is semi-open in Y. A subset $W$ of $X$ is said to be semi-compact [16] (resp. RS-compact [30], $S$-closed [29], $N$-closed [11], Quasi $H$-closed (abbreviated QHC [31]) subset relative to $X$, if for each semi-open (resp. regular semi-open, semi-open, open, open) cover $\{U \alpha: \alpha \in \nabla\}$ of $W$, there exists a finite subfamily $\nabla_{0}$ of $\nabla$ such that $W \subseteq \cup\left\{U \alpha: \alpha \in \nabla_{0}\right\}$ (resp. $W \subseteq \cup\left\{\operatorname{Int}(U \alpha): \alpha \in \nabla_{0}\right\}, W \subseteq \cup\left\{\operatorname{Cl}(U \alpha): \alpha \in \nabla_{0}\right\}, W \subseteq \cup\{\operatorname{Int}(\operatorname{Cl}(U \alpha)):$ $\left.\left.\alpha \in \nabla_{0}\right\}, W \subseteq U\left\{\mathrm{Cl}(U \alpha): \alpha \in \nabla_{0}\right\}\right)$. A space is said to be semi-compact [16] (resp. $S$-closed [33], s-closed [15]) if for each semi-open cover of the space contains a finite subcollection whose unions (resp. closures, semi-closures) cover the space. A space is said to be QHC [31] (resp. strongly compact [2], $\alpha$-compact [25], $\beta$-compact [3]) if every open (resp. preopen, $\alpha$-open, $\beta$-open) cover of the space contains a finite subcollection whose closures (resp. unions) cover the space. A space $(X, \tau)$ is called Lindelöf (resp. $S$-Lindelöf [14]) if every open (resp. semi-open) cover contains a countable subcover. A space is called lightly compact [8] if any countable open cover of the space has a finite subfamily, the closures of whose members cover the space. Recall that a space $(X, \tau)$ is resolvable [19] if there is a dense subset $D \subseteq X$ for which $X-D$ is also dense. A space which is not resolvable is called irresolvable. Spaces having only the property that their dense subsets are open are called submaximal [9]. Clearly every submaximal space is irresolvable. A space $(X, \tau)$ is called extremally disconnected (briefly E. D.) if the closure of every open set is open. A subset $W$ of a space $(X, \tau, I)$ is said to be $I$-compact [27], if for every open cover $\{U \alpha: \alpha \in \nabla\}$ of $W$, there exists a finite subcollection $\left\{U \alpha_{i}: i=1,2, \cdots, n\right\}$ such that $W-\cup\left\{U \alpha_{i}: i=1,2, \cdots, n\right\} \in I$. $(X, \tau, I)$ is said to be $I$-compact if $X$ is $I$-compact as a subset. A space $(X, \tau, I)$ is said to be countably $I$-compact or countably-compact modulo $I$ ) [27], [18] iff for every countable open cover $\{U \alpha: \alpha \in \nabla\}$ of $X$ there exists a finite subfamily 
$\left\{U \alpha_{i}: i=1,2, \cdots, n\right\}$ such that $X-\cup\left\{U \alpha_{i}: i=1,2, \cdots, n\right\} \in I$. Observe that the usual definition of countable compactness coincides with countable $\langle\phi\rangle$ compactness.

Countable $I$-compactness (countably-compact modulo $I$ ) had been studied extensively in [18] and [27].

\section{SI-Compact Spaces}

Definition 2.1. A space $(X, \tau, I)$ is said to be $S I$-compact if for every semi-open cover $\{U \alpha: \alpha \in \nabla\}$ of $X$ there exists a finite subfamily $\left\{U \alpha_{i}: i=\right.$ $1,2, \cdots, n\}$ such that $X-\cup\left\{U \alpha_{i}: i=1,2, \cdots, n\right\} \in I$.

Remark 2.1. From the above definition, we observe that:

(i) The class of SI-compactness is contained in the class of I-compactness.

(ii) A space is $S\langle\phi\rangle$-compact iff it is semi-compact.

Theorem 2.1. A space $(X, \tau)$ is semi-compact iff $\left(X, \tau, I_{f}\right)$ is $S I_{f}$-compact.

Lemma 2.1. [28] Njaistad had proved that $\mathrm{SO}(X, \tau)$ is a topology iff $(X, \tau)$ is extremally disconnected.

Lemma 2.2. [4] If $(X, \tau)$ is submaximal, and extremally disconnected, then $\tau=\mathrm{SO}(X, \tau)=\operatorname{PO}(X, \tau)=\tau^{\alpha}=\beta \mathrm{O}(X, \tau)$.

Theorem 2.2. If $(X, \tau)$ is a space. Consider the following:
(i) $(X, \tau)$ is $S\langle\phi\rangle$-compact.
(ii) $(X, \tau)$ is $S I_{f}$-compact.
(iii) $(X, \tau)$ is I-compact.
(iv) $(X, \tau)$ is compact.
(v) $(X, \tau)$ is semi-compact.
(vi) $(X, \tau)$ is strongly compact.
(vii) $(X, \tau)$ is $\alpha$-compact.
(viii) $(X, \tau)$ is $\beta$-compact.

1. If $(X, \tau)$ is E.D., then the properties from (i) to (v) are equivalent.

2. By adding the condition of submaximality then the properties from (i) to (viii) are equivalent.

The following results are immediate and the obvious proofs are omitted. 
Theorem 2.3. Let $(X, \tau, I)$ be SI-compact. If $J$ is an ideal on $X$ with $I \subseteq J$, then $(X, \tau, J)$ is $S J$-compact.

Corollary 2.1. (i) If $\left(X, \tau, I_{f}\right)$ is $S I_{f}$-compact, then $(X, \tau)$ is s-closed.

(ii) If $\left(X, \tau, I_{f}\right)$ is $S I_{f}$-compact, then $(X, \tau)$ is $S$-closed.

(iii) If $\left(X, \tau, I_{f}\right)$ is $S I_{f}$-compact, then $(X, \tau)$ is $\mathrm{QHC}$.

Theorem 2.4. Let $(X, \tau, I)$ be a space. If $I_{n} \subseteq I$ and $(X, \tau)$ is $S$-closed, then $(X, \tau)$ is SI-compact.

Proof. Immediate.

Theorem 2.5. Let $(X, \tau)$ be a space, then $(X, \tau)$ is $\operatorname{SI}_{n}$-compact iff $(X, \tau)$ is S-closed.

Proof. (Necessity) Suppose that $\{U \alpha: \alpha \in \nabla\}$ is a semi-open cover of $X$, then there exists a finite subcollection $\left\{U \alpha_{i}: i=1,2, \cdots, n\right\}$ such that $X-\cup\left\{U \alpha_{i}: i=1,2, \cdots, n\right\} \in I_{n}$ which implies to: $X-\cup\left\{\operatorname{Cl}\left(\operatorname{Int}\left(U \alpha_{i}\right)\right): i=\right.$ $1,2, \cdots, n\}=\phi$. Hence $X-\cup\left\{\operatorname{Cl}\left(\operatorname{Int}\left(U \alpha_{i}\right)\right): i=1,2, \cdots, n\right\}=\cup\left\{\operatorname{Cl}\left(U \alpha_{i}\right)\right):$ $i=1,2, \cdots, n\}$.

(Sufficiency): Let $\{U \alpha: \alpha \in \nabla\}$ be a semi-open cover of $X$, then there exists a finite subcover $\left\{U \alpha_{i} ; I=1,2, \cdots, n\right\}$ such that $X=\cup\left\{\mathrm{Cl}\left(U \alpha_{i}\right)\right)$ : $i=1,2, \cdots, n\}$, implies $X-U\left\{\operatorname{Cl}\left(\operatorname{Int}\left(U \alpha_{i}\right)\right): i=1,2, \cdots, n\right\}=\phi$., implies, $\operatorname{Int}\left(\mathrm{Cl}\left(X-\cup\left\{\left(U \alpha_{i}\right): i=1,2, \cdots, n\right\}\right)\right)=\phi$, implies, $X-\cup\left\{\left(U \alpha_{i}\right): i=\right.$ $1,2, \cdots, n\} \in I_{n}$, which complete the proof.

Theorem 2.6. If $\left(X, \tau, I_{c}\right)$ is $S I_{c}$-compact, then $(X, \tau)$ is S-Lindelöf.

Proof. Assume that $\{U \alpha: \alpha \in \nabla\}$ is a semi-open cover of $X$, then there exists a finite subfamily $\left\{\left(U \alpha_{i}\right): i=1,2, \cdots, n\right\}$ such that $X-\cup\left\{\left(U \alpha_{i}\right): i=\right.$ $1,2, \cdots, n\} \in I_{c}$ which means that $X-\cup\left\{\left(U \alpha_{i}\right): i=1,2, \cdots, n\right\}$ has a countable subcover. Hence $(X, \tau)$ is $S$-Lindelöf.

Corollary 2.2. If $\left(X, \tau, I_{c}\right)$ is $S I_{c}$-compact, then $(X, \tau)$ is Lindelöf. 
The following lemma is very useful in studying the preservation of $\mathrm{S} I$ compactness by certain types of functions.

Lemma 2.3. [17] Let $f:(X, \tau, I) \rightarrow(Y, \sigma)$ be a function. Then $f(I)=$ $\{f(E): E \in I\}$ is an ideal on $Y$.

Theorem 2.7. Let $f:(X, \tau, I) \rightarrow(Y, \sigma)$ be an irresolute surjection. If $(X, \tau)$ is $S I$-compact, then $(Y, \sigma)$ is $S f(I)$-compact.

Proof. Let $\{V \alpha: \alpha \in \nabla\}$ be a $\sigma$-semi-open cover of $Y$, then $\left\{f^{-1}(V \alpha): \alpha \in\right.$ $\nabla\}$ is a $\tau$-semi-open cover of $X$ from irresoluteness. By hypothesis, there exists a finite subcollection, $\left\{f^{-1}\left(V \alpha_{i}\right): i=1,2, \cdots, n\right\}$ such that $X-\cup\left\{f^{-1}\left(V \alpha_{i}\right)\right.$ : $i=1,2, \cdots, n\} \in I$, implies, $Y-\cup\left\{V \alpha_{i}: i=1,2, \cdots, n\right\} \in f(I)$. Therefore $(Y, \sigma)$ is $\mathrm{S} f(I)$-compact.

Theorem 2.8. Let $f:(X, \tau) \rightarrow(Y, \sigma)$ be $\beta$-irresolute from $S$ I-compact space $X$, in which every $\beta$-open set is closed into $Y$. Then $f(X)$ is $S f(I)$ compact relative to $Y$.

Proof. Is clear.

The following lemma gives a sufficient condition for the inverse image of an ideal to be an ideal.

Lemma 2.4. [17] If $f:(X, \tau) \rightarrow(Y, \sigma)$ is an injection and $J$ is an ideal on $Y$, then $f^{-1}(J)$ is an ideal on $X$.

Theorem 2.9. If $f:(X, \tau) \rightarrow(Y, \sigma, J)$ is pre-semi-open bijection and $(Y, \sigma)$ is $S J$-compact, then $(X, \tau)$ is $S f^{-1}(J)$-compact.

Proof. It is similar to the proof of Theorem 2.8 .

Theorem 2.10. Let $(X, \tau, I)$ be a space. If $\left(X, \tau^{* s}\right)$ is SI-compact, then $(X, \tau)$ is SI-compact, where $\tau^{* s}(I)$ the topology generated by the subbasis $\{U-E$ : $U \in \mathrm{SO}(X)$ and $E \in I\}[7]$. 
Proof. The result is immediate from the observation that $\tau \subseteq \tau^{* s}(I)$ (see [7]).

Theorem 2.11. Let $(X, \tau, I)$ be a space. The following are equivalent:

(i) $(X, \tau)$ is SI-compact.

(ii) For every family $\left\{F_{\alpha}: \alpha \in \nabla\right\}$ of semi-closed sets of $X$ for which $\cap\left\{F_{\alpha}: \alpha \in \nabla\right\}=\phi$, there exists a finite subfamily $\left\{F_{\alpha i}: i=1,2, \cdots, n\right\}$ such that $\cap\left\{F_{\alpha i}: i=1,2, \cdots, n\right\} \in I$.

Proof. To show that (i) implies (ii) Let $\left\{F_{\alpha}: \alpha \in \nabla\right\}$ be a family of semi closed sets for which $\cap\left\{F_{\alpha}: \alpha \in \nabla\right\}=\phi$. Then $\left\{X-F_{\alpha}: \alpha \in \nabla\right\}$ is a semi-open cover of $X$, by (i), there exists a finite subcollection $\left\{X-F_{\alpha i}: i=1,2, \cdots, n\right\}$ such that $X-\cup\left\{\left(X-F_{\alpha i}\right): i=1,2, \cdots, n\right\} \in I$. Hence $\cap\left\{F_{\alpha i}: i=1,2, \cdots, n\right\} \in$ I.

(ii) implies (i): Suppose that $\{U \alpha: \alpha \in \nabla\}$ is a semi-open cover of $X$. Then $\{X-U \alpha: \alpha \in \nabla\}$ is a collection of semi-closed sets and $\cap\{(X-U \alpha): \alpha \in$ $\nabla\}=\phi$. Hence there exists a finite subcollection $\left\{\left(X-U_{\alpha i}\right) ; i=1,2, \cdots, n\right\}$ such that $\cap\left\{\left(X-U_{\alpha i}\right): i=1,2, \cdots, n\right\} \in I$. From De Morgan's Law, we have $X-\cup\left\{\left(U_{\alpha i}\right): i=1,2, \cdots, n\right\} \in I$. Thus $(X, \tau)$ is SI-compact.

Remark 2.2. If $I=\{\phi\}$ in the previous theorem, we obtain the standard characterizations of semi compactness.

Theorem 2.12. If $(X, \tau, I)$ is SI-compact, then for every cover $\{U \alpha$ : $\alpha \in \nabla\}$ of regular closed sets of $X$, there exists a finite subfamily $\left\{U_{\alpha i}: i=\right.$ $1,2, \cdots, n\}$. Such that $X-\cup\left\{U_{\alpha i}: i=1,2, \cdots, n\right\} \in I$.

Proof. Follows from the fact that each regular closed set is semi-open set.

Theorem 2.13. For a space $(X, \tau, I)$, if $(X, \tau)$ is SI-compact, then any preopen cover $\{U \alpha: \alpha \in \nabla\}$ of $X$ has a finite subcover $\left\{U_{\alpha i}: i=1,2, \cdots, n\right\}$. Such that $X-\cup\left\{\mathrm{Cl}\left(U_{\alpha i}\right): i=1,2, \cdots, n\right\} \in I$.

Proof. Follows directly from the fact that, the closure of each preopen set 
is semi-open.

Corollary 2.3. If $(X, \tau, I)$ is SI-compact, then any preclosed family $\left\{P_{\alpha}\right.$ : $\alpha \in \nabla\}$ for which $\cap\left\{P_{\alpha}: \alpha \in \nabla\right\}=\phi$, has a finite subfamily $\left\{P_{\alpha i}: i=\right.$ $1,2, \cdots, n\}$ such that $\cap\left\{\left(\operatorname{Int}\left(P_{\alpha i}\right): i=1,2, \cdots, n\right\} \in I\right.$.

Lemma 2.5. [28] If $(X, \tau)$ is a space, then: $\operatorname{SO}(X, \tau)=\operatorname{SO}\left(X, \tau^{\alpha}\right)$.

Theorem 2.14. A space $(X, \tau, I)$ is SI-compact iff $\left(X, \tau^{\alpha}, I\right)$ is SI-compact.

Proof. Let $\{U \alpha: \alpha \in \nabla\}$ be a $\tau^{\alpha}$-semi-open cover of $X$. Then there exists a finite subfamily $\left\{U_{\alpha i}: i=1,2, \cdots, n\right\}$ such that $X-\cup\left\{U_{\alpha i}: i=1,2, \cdots, n\right\} \in I$ (Lemma 2.5). Hence, $\left(X, \tau^{\alpha}\right)$ is $S I$-compact. The converse is obvious.

Theorem 2.15. The property of being SI-compact is semi-topological property.

Proof. Obvious from lemma 2.5.

Lemma 2.6. [5]. If $A$ is $\tau$-dense and $\tau$-semi-open, then $\operatorname{SO}(X, \tau)=$ $\mathrm{SO}(X, \tau(A))$.

Theorem 2.16. A space $(X, \tau, I)$ is SI-compact iff $(X, \tau(A), I)$ is $S I$ compact, where $A$ is $\tau$-dense and $\tau$-semi-open.

Proof. Follows directly from Lemma 2.6.

In 1969, J. S. Miodnszewski, et.al [26] proved the following lemma.

Lemma 2.7. If $\tau \subseteq \tau^{\prime}$, then $\mathrm{RO}(X, \tau)=\mathrm{RO}\left(X, \tau^{\prime}\right)$ iff $\mathrm{Cl}_{\tau}(W)=\mathrm{Cl}_{\tau^{\prime}}(W)$ for every $W \in \tau^{\prime}$.

By making use of Lemma 2.7, we introduce the following result.

Corollary 2.4. For a space $(X, \tau)$ and $A \subseteq X$, we have: $\operatorname{RO}(X, \tau)=$ $\mathrm{RO}(X, \tau[A])$, consequently, $\mathrm{RC}(X, \tau)=\mathrm{RC}(X, \tau[A])$, if $A \cap U=\phi$ for every $U \in \tau$ and $U \neq X$. 
Theorem 2.17. If $(X, \tau)$ is SI-compact, then $(X, \tau[A])$ is SI-compact, if $A \cap U=\phi$, for every $U \in \tau$ and $U \neq X$.

Proof. Obvious; by using Corollary 2.4 .

Lemma 2.8. [6]. If $(X, \tau)$ is an irresolvable space, $\tau(\Im)$ is a filter extension of $\tau$ by a filter $\Im$ on $X$ and $F \in \mathrm{SO}(X, \tau)$, for every $\mathbb{F} \in \Im$, then $\operatorname{SO}(X, \tau)=$ $\mathrm{SO}(X, \tau(\Im))$.

Theorem 2.18. If $(X, \tau, I)$ is SI-compact and $\tau(\Im)$ is a filter extension of $\tau$ by a filter $\Im$ on $X$, then $(X, \tau(\Im), I)$ is SI-compact iff $(X, \tau)$ is irresolvable and $\mathbb{F} \in \operatorname{SO}(X, \tau)$ for every $\mathbb{F} \in \Im$.

Proof. Let $\{U \alpha: \alpha \in \nabla\}$ be a semi-open cover of $X$ by $\tau(\Im)$-semi-open sets. Then $\{U \alpha: \alpha \in \nabla\}$ is a $\tau$-semo-open cover, Since $(X, \tau)$ is irresolvable (Lemma 2.8) and $\mathbb{F} \in \mathrm{SO}(X, \tau)$ for every $\mathbb{F} \in \Im$, since $(X, \tau)$ is $S I$-compact, there exists a finite subcollection $\left\{U_{\alpha i}: i=1,2, \cdots, n\right\}$ such that, $X-\cup\left\{U_{\alpha i}\right.$ : $i=1,2, \cdots, n\} \in I$. Hence $(X, \tau(\Im), I)$ is SI-compact.

\section{SI-Compact Subsets Relative to a Space}

Definition 3.1. A subset $W$ of a space $(X, \tau, I)$ is said to be $S I$-compact relative to $X$ if for every semi-open cover $\left\{U_{\alpha}: \alpha \in \nabla\right\}$ of $W$, there exists a finite subcollection $\left\{U_{\alpha i}: i=1,2, \cdots, n\right\}$ such that $W-\cup\left\{U_{\alpha i}: i=1,2, \cdots, n\right\} \in I$.

Theorem 3.1. The following are equivalent for a subset $W$ of $X$.

(i) $W$ is $S I_{f}$-compact relative to $X$.

(ii) $W$ is $S\langle\phi\rangle$-compact relative to $X$.

(iii) $W$ is semi-compact relative to $X$.

Theorem 3.2. For a subset $W$ of an E. D. space $X$, the following are equivalent:

(i) $W$ is $S I_{f}$-compact relative to $X$.

(ii) $W$ is $R S$-compact relative to $X$. 
(iii) $W$ is $S$-closed relative to $X$.

(iv) $W$ is $N$-closed relative to $X$.

(v) $W$ is $Q H C$ relative to $X$.

Theorem 3.3. If $W_{i}, i=1,2$, are SI-compact sets relative to a space $(X, \tau, I)$, then $W_{1} U W_{2}$ is SI-compact set reltaive to $X$.

Corollary 3.1. The intersection of two open sets having SI-compact complement is also open having SI-compact complement.

Theorem 3.4. Let $(X, \tau)$ be a space with an ideal $I$ an $X$ and $W \in \tau^{\alpha}$. W is SI-compact iff $(W, \tau \mid W)$ is $S I \mid W$-compact.

Proof. (Necessity) Let $\left\{U_{\alpha} \cap W\right\}$ be $\tau \mid W$-semi-open cover of $W$, where $U_{\alpha} \in \operatorname{SO}(X, \tau)$ for each $\alpha$. Now $\left\{U_{\alpha}\right\}$ is a $\tau$-semi-open cover of $W$ and hence there exists a finite subfamily $\left\{U_{\alpha i}\right\}$ such that $\left.\mathbb{W}-U\left\{U \alpha_{i}: i=1,2, \cdots, n\right\}\right) \in$ I. This implies $\mathbb{W} \cap\left(\mathbb{W}-\cup\left\{U_{\alpha i}: i=1,2, \cdots, n\right\}\right) \in I \cap \mathbb{W}$, and we have $W \cap\left(W-\cup\left\{U \alpha_{i}: i=1,2, \cdots, n\right\}\right)=W-\cup\left\{\left(\mathbb{W} \cap U \alpha_{i}\right): i=1,2, \cdots, n\right\} \in I \mid W$. Thus, $(W, \tau \mid W)$ is $S I \mid W$-compact.

(Sufficiency) Let $\{U \alpha: \alpha \in \nabla\}$ be a $\tau$-semi-open cover of $W$. Then $\{U \alpha \cap W\}$ is $\tau \mid W$ semi-open cover of $W$. There exists then a finite subfamily $\left\{U_{\alpha i} \cap \mathbb{W}\right\}$ such that $W-\cup\left\{U_{\alpha i} \cap W: i=1,2, \cdots, n\right\} \in I \mid W \subseteq I$. Hence $W$ is SI-compact.

Theorem 3.5. A subset $W$ of a space $(X, \tau, I)$ is SI-compact relative to $X$, if for every cover $\{U \alpha: \alpha \in \nabla\}$ of $W$ such that $U \alpha \in \beta O(X)$, there exists a finite subcollection $\left\{U_{\alpha i}: i=1,2, \cdots, n\right\}$ such that $W-\cup\left\{\mathrm{Cl}\left(U_{\alpha i}\right): i=1,2, \cdots, n\right\} \in$ I.

Proof. It is similar to the proof of Theorem 2.13. 


\section{Countably SI-Compact Spaces}

Definition 4.1. A space $(X, \tau, I)$ is said to be countably SI-compact or countably S-compact modulo $I$, iff for every countable semi-open cover $\{U \alpha$ : $\alpha \in \nabla\}$ of $X$, there exists a finite subfamily $\left\{U_{\alpha i}: i=1,2, \cdots, n\right\}$ such that $X-\cup\left\{U_{\alpha i}: i=1,2, \cdots, n\right\} \in I$.

Remark 4.1. From the above definition, we observe that:

1. The ordinary definition of S-Lindelöf coincides with countable $S\langle\phi\rangle$-compactness.

2. Every $S I$-compact space is countably $S I$-compact.

3. A space $(X, \tau)$ is $\mathrm{S}$-Lindelöf iff it is countable $S I_{f}$-compact.

4. Every countably SI-compact is countably $I$-compact.

Theorem 4.1. If $(X, \tau, I)$ is countably SI-compact and $J$ is an ideal on $X$ such that $I \subseteq J$. Then $(X, \tau, J)$ is countably $S J$-compact.

Theorem 4.2. If $(X, \tau, I)$ is countably SI-compact and S-Lindelöf, then $(X, \tau, I)$ is SI-compact.

Definition 4.2. [27] Given a space $(X, \tau, I), I$ is called $\tau$ boundary if $\tau \cap I=\{\phi\}$.

Theorem 4.3. If $(X, \tau, I)$ is countably SI-compact and I is $\tau$ boundary, then $(X, \tau)$ is lightly compact.

Proof. Assume that $\left\{U_{\alpha}: \alpha \in \nabla\right\}$ is a countable open cover of $X$, then it is semi-open cover, there exists a finite subcollection $\left\{U_{\alpha i}: i=1,2, \cdots, n\right\}$ such that $X-\cup\left\{U_{\alpha i}: i=1,2, \cdots, n\right\}=E \in I$. Since $I$ is $\tau$-boundary, then $\operatorname{Int}(E)=\phi$, implies, $\operatorname{Int}\left(X-\cup\left\{U_{\alpha i}: i=1,2, \cdots, n\right\}\right)=\operatorname{Int}(E)=\phi$, implies $X=\cup\left\{\mathrm{Cl}\left(U_{\alpha_{i}}\right): i=1,2, \cdots, n\right\}$. Therefore $(X, \tau)$ is lightly compact.

Theorem 4.4. The following are equivalent for a space $(X, \tau, I)$.

(i) $(X, \tau)$ is countably SI-compact. 
(ii) For every countable family $\left\{F_{\alpha}: \alpha \in \nabla\right\}$ of semi-closed sets such that $\cap\left\{F_{\alpha}\right.$ : $\alpha=1,2, \cdots, \infty\}=\phi$. There exists a finite subfamily $\left\{F_{\alpha i}: i=1,2, \cdots, n\right\}$ such that $\cap\left\{F_{\alpha i}: i=1,2, \cdots, n\right\} \in I$.

Proof. It is similar to the proof of Theorem 2.11 .

Corollary 4.1. If $(X, \tau)$ is countably SI-compact then for every countable family $\left\{F_{\alpha}: \alpha \in \nabla\right\}$ of regular closed sets such that $\cap\left\{F_{\alpha}: \alpha=1,2, \cdots, \infty\right\}=\phi$ there exists a finite subfamily $\left\{F_{\alpha i}: i=1,2, \cdots, n\right\}$ such that $\cap\left\{F_{\alpha i}: i=\right.$ $1,2, \cdots, n\} \in I$.

The obvious proofs of the following theorems are omitted.

Theorem 4.5. Let $f:(X, \tau, I) \rightarrow(Y, \sigma)$ be irresolute surjection. If $(X, \tau, I)$ is countably $S I$-compact, then $(Y, \sigma)$ is countably $S f(I)$-compact.

Theorem 4.6. If $f:(X, \tau) \rightarrow(Y, \sigma, J)$ is pre-semi-open bijection and $(Y, \sigma)$ is countably $S J$-compact, then $(X, \tau)$ is countably $S f^{-1}(J)$-compact.

\section{References}

[1] M. E. Abd El-Monsef; S. N. El-Deeb and R. A. Mahmoud, " $\beta$-open sets and $\beta$-continuous mappings", Bull. Fac. Sci., Assiut Univ., 12 (1) (1983), 77-90.

[2] M. E. Abd El-Monsef; I. A. Hasanein; A. S. Mashhour and T. Noiri, "Strongly compact spaces", Delta J. Sci., (8) (1984), 30-46.

[3] M. E. Abd El-Monsef and A. M. Kozae, "Some generalized forms of compactness and closedness", Delta J. Sci., (9) (2) (1985), 257-269.

[4] _ _ "On extremally disconnectedness ro-equivlence and properties of some maximal topologies", Proc. $4^{\text {th }}$ Conf. Oper. Res. and Math. Method, Alex. Univ., (1988).

[5] M. E. Abd El-Monsef and E. F. Lashien, "On Simple Extensions of topologies", Journal of the Fac. of Edu., Ain Shams Univ., Fifth Top. Symp., No 12 (1987), 71-82.

[6] M. E. Abd El-Monsef and E. F. Lashien, "Filter Extensions of Topologies", accepted for

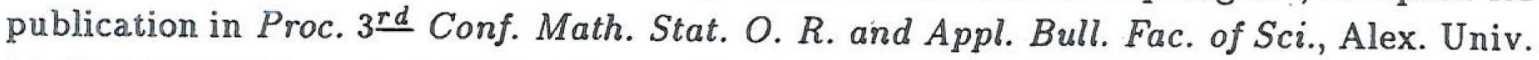

[7] M. E. Abd El-Monsef, E. F. Lashien and A. A. Nasef, "Some Topological Operators Via Ideals", to appear in the First International Conference on Topology and Its Applications in 1990.

[8] R. W. Bagley, E. H. Connell, and J. D. McKnight, "On properties charactrizing pseudo compact spaces", Proc. Amer. Math. Soc., 9 (1958), 500-506.

[9] N. Bourbaki, "General Topology", Addison-Wesley, Mass., 1966.

[10] D. E. Cameron, "Properties of S-closed spaces", Proc. Amer. Math. Soc., V. (72), (3) (1978), 581-586. 
[11] D. Carnahan, "Locally nearly-compact spaces", Bull. Un. Math. Ital., (4) 6 (1972), 146153.

[12] S. G. Grossley and S. K. Hildebrand, "Semi-closure", Texas J. Sci., $22(2+3)$ (1971), 99-112.

[13] ___, "Semi-Topological properties", Fund. Math., 74 (1972), 233-254.

[14] G. Di Maio, "S-closed spaces, S-sets and S-continuous functions", Atti-Accad. Sci. Torino, 118 (1984), 125-134.

[15] G. Di Maio and T. Noiri, "On s-closed spaces", Indian J. Pure Appl. Math., 18 (3) (1987), 226-233.

[16] C. Dorsett, "Semi convergence and semi-compactness", Indian J. Math. Mech., 19 (1981), 11-17.

[17] T. R. Hamlett and D. Janković, "Compactness with respect to an ideal", Boll. U. M. I., (7), 4-B (1990), 849-861.

[18] T. R. Hamlett, D. Jankovic and D. Rose, "Countable compactness with respect to an ideal Math", Chron., 20 (1991), 109-126.

[19] E. Hewitt, "A problem of set theoretic topology", Duke Math. J., 10 (1943), 309-333.

[20] K. Kuratowski, "Topologies I", Warszawa, 1993.

[21] N. Levine, "Semi-open sets and semi-continuity in topological spaces", Amer. Math. Monthly, 70 (1963), 36-41.

[22] N. Levine, "Simple extensions of topologies", Amer. Math. Monthly, 71 (1964), 22-25.

[23] R. A. Mahmoud and M. E. Abd El-Monsef, " $\beta$-Irresolute and $\beta$-Topological invariant", accepted in Pakistan Academy of Sciences in 1990.

[24] A. S. Mashhour, M. E. Abd El-Monsef and S. N. El-Deeb, "On precontinuous and weak precontinuous mappings", Proc. Math. Phys. Soc., Egypt. (53) (1982), 47-53.

[25] A. S. Mash hour, I. A. Hasanein and S. N. El-Deeb, " $\alpha$-continuous and $\alpha$-open mappings", Acta, Math. Acad. Sci. Hungar, (3+4) (1983), 213-218.

[26] J. Mioduszewski and L. Rudolf, "H-closed and extremally disconnected Hausdörff spaces", Dissertatias Math., 66 (1969).

[27] R. L. Newcomb, "Topologices which are compact modulo an ideal", Ph. D. dissertation, Univ. of Cal. at Santa Barbara, 1967.

[28] O. Njåstad, "On some classes of nearly open sets", Pacific J. Math., 15 (1965), 961-970.

[29] T. Noiri. "On S-closed spaces", Ann. Soc. Sci. Bruxelles, 91 (1977), 189-194.

[30] __ "On RS-compact spaces", J. Korean Math. Soc., 22 (1985), No 1, pp. 19-34.

[31] J. Porter and J. Thomas, "On H-closed and minimal Hausdörff spaces", Trans. Amer. Math. Soc., 138 (1969), pp. 159-170.

[32] D. V. Rančin, "Compactness modulo an ideal", soviet Math. Dokl, Vol (13) (1972), No. 1, $193-197$.

[33] T. S. Thompson, "S-closed spaces", Proc. Amer. Math. Soc., 60 (1976), 335-338.

[34] S. P. Young, "Local discrete extensions of topologies Kyungpook", Matiı. J., 11 (1971), $21-24$,

Tanta University, Tanta, Egypt. 